\title{
Patterns of gene evolution following duplications and speciations in vertebrates
}

\author{
Kyle T. David ${ }^{\text {Corresp., } 1}{ }^{1}$ Jamie R. Oaks ${ }^{1}$, Ken M. Halanych ${ }^{1}$ \\ ${ }^{1}$ Department of Biological Sciences, Auburn University, Auburn, Alabama, United States \\ Corresponding Author: Kyle T. David \\ Email address: kzd0038@auburn.edu
}

\section{Background}

Eukaryotic genes typically form independent evolutionary lineages through either speciation or gene duplication events. Generally, gene copies resulting from speciation events (orthologs) are expected to maintain similarity over time with regard to sequence, structure, and function. After a duplication event, however, resulting gene copies (paralogs) may experience a broader set of possible fates, including partial (subfunctionalization) or complete loss of function, as well as gain of new function (neofunctionalization). This assumption, known as the Ortholog Conjecture, is prevalent throughout molecular biology and notably plays an important role in many functional annotation methods.

Unfortunately, studies that explicitly compare evolutionary processes between speciation and duplication events are rare and conflicting.

\section{Methods}

To provide an empirical assessment of ortholog/paralog evolution, we estimated ratios of nonsynonymous to synonymous substitutions $(\omega=\mathrm{dN} / \mathrm{dS}$ ) for 251,044 lineages in 6,244 gene trees across 77 vertebrate taxa.

\section{Results}

Overall, we found $\omega$ to be more similar between lineages descended from speciation events $(p<0.001)$ than lineages descended from duplication events, providing strong support for the Ortholog Conjecture. The asymmetry in $\omega$ following duplication events appears to be largely driven by an increase along one of the paralogous lineages, while the other remains similar to the parent. This trend is commonly associated with neofunctionalization, suggesting that gene duplication is a significant mechanism for generating novel gene functions. 


\section{Patterns of Gene Evolution Following Duplications and Speciations in Vertebrates}

2

4

6

7 Corresponding Author:

8 Kyle David

9101 Rouse Life Sciences Building, Auburn, Alabama, 36849

10 Email address: kzd0038@auburn.edu

\section{Abstract}

\section{Background}

Eukaryotic genes typically form independent evolutionary lineages through either speciation or gene duplication events. Generally, gene copies resulting from speciation events (orthologs) are expected to maintain similarity over time with regard to sequence, structure, and function. After a duplication event, however, resulting gene copies (paralogs) may experience a broader set of possible fates, including partial (subfunctionalization) or complete loss of function, as well as gain of new function (neofunctionalization). This assumption, known as the Ortholog Conjecture, is prevalent throughout molecular biology and notably plays an important role in many functional annotation methods. Unfortunately, studies that explicitly compare evolutionary processes between speciation and duplication events are rare and conflicting.

\section{Methods}

To provide an empirical assessment of ortholog/paralog evolution, we estimated ratios of nonsynonymous to synonymous substitutions $(\omega=\mathrm{dN} / \mathrm{dS})$ for 251,044 lineages in 6,244 gene trees across 77 vertebrate taxa.

\section{Results}

Overall, we found $\omega$ to be more similar between lineages descended from speciation events $(p<0.001)$ than lineages descended from duplication events, providing strong support for the Ortholog Conjecture. The asymmetry in $\omega$ following duplication events appears to be largely driven by an increase along one of the paralogous lineages, while the other remains similar to the parent. This trend is commonly associated with neofunctionalization, suggesting that gene duplication is a significant mechanism for generating novel gene functions. 


\section{Introduction}

36 Homologous relationships between eukaryotic genes are typically categorized as either

37 orthologous or paralogous. Orthologs are gene copies that arise through speciation events and paralogs are gene copies that arise through duplication events (Fig. 1). The distinction between orthologs and paralogs has important implications for molecular biology largely due to the assumption that orthologs maintain similarity over time as genes are expected to serve comparable roles in descendant species ${ }^{1-3}$. By contrast, a much wider range of fates are considered for paralogs ${ }^{4-6}$. In his seminal work, $\mathrm{Ohno}^{7}$ hypothesized several possible fates for duplicated genes. Paralogs may maintain their ancestral function (conservation ${ }^{7}$ ), gain new function (neofunctionalization ${ }^{8}$ ), divide or specialize the ancestral function between copies $\left(\right.$ subfunctionalization $^{8}$ ), or lose function entirely. Many additional models have since been proposed but which broadly fall under these main categories $5,6,9,10$. These include escape from adaptive conflict ${ }^{11,12}$, under which genes gain new function while continuing to perform the ancestral function as well as several dosage models, under which genes are preferentially retained or conserved on the basis of dosage effects.

The expectation of conservation between orthologs and divergence between paralogs is sometimes referred to as the "Ortholog Conjecture"13,14; an assumption so pervasive throughout genomics that it is not always referenced explicitly. For example, the Ortholog Conjecture is often used indirectly to infer gene function ${ }^{2,13}$. Under this method, the known function of a gene from Mus musculus would be assumed as the function for orthologs in other species where the gene has not been functionally characterized. Many popular online databases such as $\mathrm{KEGG}^{15}$, PANTHER $^{16}$, and eggNOG ${ }^{17}$ rely on evidence from orthology to assign function. Additionally, as of October 2019, there are 386,841 proteins with orthology evidence in the Swiss-Prot sequence database representing $70 \%$ of all entries ${ }^{18}$.

Given the importance of the Ortholog Conjecture in biology, the fact that only few studies test it explicitly (reviewed in ${ }^{3,19}$ ) is surprising. A preliminary study using microarrays between human and mouse recovered significant differences in expression profiles between ortholog pairs, at levels comparable with gene pairs selected at random ${ }^{20}$. However, when these data were reanalyzed ${ }^{21}$, orthologs were found to be significantly more similar to one another than paralogs. Nehrt et. $\mathrm{al}^{22}$ used Gene Ontology annotations and found no support for the Ortholog 
65 Conjecture between humans and mice, however other researchers have since noted several 66 pitfalls associated with using Gene Ontology ${ }^{23-26}$. Recently, a study by Kryuchkova-Mostacci 67 and Robinson-Rechavi ${ }^{27}$ found strong support for the Ortholog Conjecture; however, reanalysis

68 by Dunn, Zapata et al. ${ }^{28}$ found the observed differences to be an artifact of node age, not the 69 divergence events themselves.

70 To address the validity of ortholog conjecture, we estimated the ratio of nonsynonymous

71 to synonymous nucleotide substitution rates $(\omega=\mathrm{dN} / \mathrm{dS})$ for daughter lineages descended from 72 inferred speciation events (orthologous lineages) or duplication events (paralogous lineages). We 73 then took the absolute difference of $\omega(\Delta \omega)$ between daughter lineages for an estimate of the 74 difference in selective pressure experienced by daughter lineages (Fig. 1). If nonsynonymous 75 substitutions are more likely between paralogs, we would expect to see greater $\Delta \omega$ values 76 following duplication events compared to speciation events (Fig. 1) $)^{3}$. We also measured the 77 difference in $\omega$ of each daughter lineage from the parent $\left(\Delta \omega_{p}\right)$ to see if one or both lineages 78 diverged from the ancestral ratio. $\Delta \omega$ was estimated for 234,066 speciation events and 16,978 79 duplication events in 6,244 gene families across 77 vertebrates (Table S1).

80

\section{Materials \& Methods}

82 We drew from 22,340 publicly available protein trees from the EnsemblCompara online database 83 (release 90$)^{29,30}$. To avoid issues with unknown calibration dates and branch lengths, we focused 84 on patterns within vertebrates for 77 target taxa and 6 outgroup taxa with a well-established 85 evolutionary history (Table S1). Calibration dates taken from the most recent literature (Table S2) were assigned to 55 nodes in the species tree and implemented in a global clock model ${ }^{31}$. Under the global clock model one rate is used for each tree, represented in millions of years. Calibrating the data in this way enables us to compare nodes from different trees to one another, even though they likely have different rates of substitution. Trees that lacked at least one node with a calibration date $(9,528)$ were discarded. Paralogs and orthologs were inferred using the species overlap algorithm ${ }^{32}$. Duplication labels were applied to nodes where the same species is represented in both daughter clades at least once. Speciation labels were then those nodes with no species overlap between the two daughter lineages. Put differently, speciation nodes gave rise to two discrete monophyletic groups, and duplication nodes did not (Fig. 1). Speciation and 
96 EnsemblCompara ${ }^{29}$, with which they were congruent in $97.5 \%$ of cases. This study ignores

97 horizontal gene transfer events and their resultant xenologs, which are unlikely between 98 vertebrates.

Trees with greater than approximately 170 tips $(\sim 5,000$ trees $)$ were found to be too

100

101

102

103

104

105

106

107

108

109

110

111

112

113

114

115

116

117

118

119

120

121

122

123

124

125

126 computationally intensive and excluded from our analyses. We also filtered 2,766 trees that did not contain at least one duplication and at least one speciation event. Of the remaining 6,303 trees, we filtered 31,039 nodes with an expected number of synonymous substitutions greater than 2 in either daughter lineage, which indicate saturation of substitutions, then 75,427 nodes with an expected number of synonymous substitutions less than 0.01 in either daughter lineage, which can lead to poor estimates of $\omega$, and finally 61 nodes with $\omega>10$ in one or both daughter lineages as outliers ${ }^{33}$. Our filtered dataset contains 6,244 trees with 16,978 duplication nodes and 234,066 speciation nodes.

We inferred selective pressure by estimating the rate of nonsynonymous substitutions relative to the rate of synonymous substitutions $(\omega)^{34}$. We estimated $\omega$ values with codeml, a maximum-likelihood method for codon-substitutions model within the PAML package ${ }^{31}$. To estimate $\Delta \omega$, we used a free-ratios model which allows separate $\omega$ values to be calculated for each branch in the tree ${ }^{35,36}$. The difference in selective pressure was then quantified by simply taking the absolute difference between the $\omega$ of the two daughter lineages $(\Delta \omega)$ as well as the difference of one daughter lineage from the parent lineage $\left(\Delta \omega_{\mathrm{p}}\right)$ for each speciation and duplication node in our tree (Fig. 1). Per the PAML authors' recommendation we first ran a null model, which assumes uniform $\omega$ values to generate branch lengths and transition/transversion ratios in an effort to limit free parameters. All analyses were performed on the High Performance Computing Hopper Cluster at Auburn University.

To effectively test the ortholog conjecture, we compared empirical values to a null model, in which there was no difference between speciation and gene duplication events. For our null model, we assigned speciation and duplication labels randomly without replacement for each gene tree, removing any putative link between evolutionary events and $\Delta \omega$. To calculate $p-$ values, we performed a two-tailed permutation test comparing our empirical estimates to those calculated from trees with permuted speciation/duplication node labels. Null distributions were approximated with 1,000 permutations. Under either model, the number of speciation and gene duplication nodes for each tree was kept the same as in the empirical trees. We also include 
127 Hedges' $g^{37}$ as a measure of effect size.

128 For our analysis, we were interested in testing not just differences between means but 129 also similarity of distributions. Considering this, we calculated the overlap coefficient (OVL) 130 between kernel density distributions of orthologous and paralogous lineages (Fig. 2A). The OVL 131 is the area in common under two probability density functions and represents the sum of the 132 conditional misclassification probability as well as providing an intuitive measure of agreement 133 between two similar distributions ${ }^{38}$. Under the most extreme interpretation of the ortholog 134 conjecture, we would expect no overlap in $\Delta \omega$ distributions between orthologous and paralogous 135 lineages. OVLs were calculated by integrating the area under the intersection between the two 136 density plots. We also perform a two-sample Kolmogorov-Smirnov test, which is a 137 nonparametric test which estimates the likelihood of two samples (in this case $\Delta \omega$ values 138 between speciation and duplication events) being drawn from the same distribution ${ }^{39}$. 139 All code required to update experiments and reproduce results/figures are available at 140 https://github.com/KyleTDavid/OrthologConjecture2019. Original data files are available at 141 https://figshare.com/projects/OrthologConjecture2019/63935.

\section{Results}

144 On average, $\Delta \omega$ was significantly smaller between orthologous lineages than paralogous lineages $145(\mathrm{p}<0.001, \mathrm{~K}-\mathrm{S}$ test $\mathrm{p}<2.2 \mathrm{E}-16$, Hedges' g=0.94) (Fig. 2A). After a speciation event, resulting 146 orthologs experience more similar patterns of molecular evolution (average $\Delta \omega$ was $0.20 \pm 0.38$ 147 standard deviations) to one another than do paralogs after a duplication event (0.60 \pm 0.77$)$. The 148 OVL of kernel density distributions of $\Delta \omega$ was $57.6 \%$ between orthologous and paralogous

149 lineages. Small but significant $(\mathrm{p}<0.001$, Hedges' $\mathrm{g}=0.18)$ differences in $\Delta \omega$ were also recovered 150 between different categories of duplication events, with greater $\Delta \omega$ between duplication events 151 leading to within-species paralogs (i.e., nodes in which all descendant leaves belong to the same 152 species $)(0.62 \pm 0.77)$ than between-species paralogs (i.e., duplication nodes in which descendant 153 leaves belong to more than one species) $(0.48 \pm 0.77)$ (Fig. S1).

154 We observe significant ( $\mathrm{p}<0.001$, Hedges' $\mathrm{g}=0.88$ ) differences in the $\omega$ ratios themselves 155 with an average ratio of $0.22 \pm 0.36$ for orthologous lineages compared to $0.56 \pm 0.70$ for 156 paralogous lineages (Fig. S2). High $\omega$ following gene duplications events is a well-documented 157 phenomenon ${ }^{9,40-42}$. However whether or not relaxed selective pressures are experienced equally 
158 between copies remains controversial ${ }^{43,44}$. To address this we also estimated the minimum and 159 maximum difference between $\omega$ of each daughter lineage per pair and their parent lineage $\left(\Delta \omega_{\mathrm{p}}\right)$

160 (Fig. 1). $\omega$ for paralogous lineages increased by $0.21 \pm 0.82$ compared to their parent lineage, 161 significantly $(\mathrm{p}<0.001$, Hedges' $\mathrm{g}=0.48)$ higher than $\omega$ for orthologous lineages which decrease 162 by $0.003 \pm 0.42$. Although differences from the parent lineage were not recovered between the 163 minimum orthologous and paralogous daughter lineages $(p=1.00)$ they were recovered for the 164 maximum lineages ( $<<0.01$ ) (Fig. 2B). Additionally, effect sizes between the maximum and 165 minimum lineages were $>100 x$ greater between paralogous (Hedges' $g=0.85$ ) and orthologous 166 lineages (Hedges' $g=6.7 \mathrm{E}-3$ ).

167

\section{Discussion}

169 Our results demonstrate support for the Ortholog Conjecture with regard to $\omega$. The relative rates 170 of nonsynonymous substitutions differs more between paralogous lineages than between 171 orthologous lineages. Our observation of higher $\Delta \omega$ in paralogous lineages appears to be largely 172 the result of a higher relative rate of nonsynonymous substitutions in just one daughter paralog 173 (Fig. 2B). This pattern is commonly thought to be indicative of Ohno's neofunctionalization ${ }^{7}$ or 174 Francino's adaptive radiation ${ }^{45}$ models of evolution ${ }^{5,10}$, indicating that diversification may be the 175 most common fate for at least one retained paralog copy. This interpretation is congruent with 176 previous studies ${ }^{42,46,47}$, such as Brunet et al. $2006^{48}$ who also observed conserved vs. elevated 177 selection between paralogous lineages resulting from the teleost specific whole genome 178 duplication event. However, a later study ${ }^{49}$ using a more rigorous model found evidence for high 179 levels of selection across lineages throughout vertebrates, regardless of homologous 180 relationships. It worth noting that this interpretation only extends insofar as relative rates of 181 substitution can be understood as a proxy for functional change. In reality the relationship 182 between in sequence, structure, and function is far from direct. For example, there is also a 183 negative correlation between $\omega$ and level of expression ${ }^{50}$. If observed $\omega$ values were driven by expression our results could also support a specialization model of subfunctionalization, under which the new copy continues to perform the ancestral function in a reduced, specialized capacity (expressed only in particular tissues, under certain environmental conditions, etc.).

Most gene duplication events in our dataset occur along lineages for which there is no 
189

190

191

192

193

194

195

196

197

198

199

200

201

202

203

204

205

206 207 lineages.

208

209

210

211

212

213

214

215

216

217

218

not more, pronounced in single/several gene duplication events (such as those produced through unequal crossing-over) than whole genome duplication events. New gene copies generated through whole genome duplication are identical in terms of just not their sequence but location and context within the (sub)genome as well ${ }^{3}$. By contrast retrotransposed gene duplicates are relocated to an entirely new genomic environment in which it will be unlikely to reproduce the ancestral function and thus free to acquire new mutations. This scenario is supported by Han et al. $2009^{42}$ who found that gene duplicates transferred to a new position are more likely to experience elevated $\omega$ ratios.

There were several clades in which the ortholog conjecture was not supported $(\mathrm{p}>0.05)$, namely: Sarcopterygii, Neopterygii, Amniota, and Mammalia. This suggests an unexpected trend of decreasing support for the ortholog conjecture as time since divergence increases (Fig. 3). This trend is likely the result of saturation in substitutions ${ }^{51}$ over time as the expected number of synonymous substitutions begin to plateau at $\sim 150$ mya while the expected number of nonsynonymous substitutions continue to increase, obscuring differences between duplication and speciation events and increasing the overlap between the distributions (Fig. S3). Additionally, estimates of $\omega$ have been demonstrated to exhibit slight bias when divergence levels are $l o w^{52}$. As a result, more work may need to be done in order to more definitively resolve questions of ortholog and paralog evolution along especially old or young vertebrate

A similar pattern was recovered between different classifications of paralogs, with slightly smaller $\Delta \omega$ for between-species paralogs than within-species paralogs (Fig S1.). This finding disagrees with previous results which suggest more conservation between within-species paralogs than between-species paralogs 22,24 . This discrepancy may be the result of saturation as noted above, as within-species duplication events are necessarily concentrated toward the tips of a phylogeny. However, when all nodes were filtered to those $<1$ mya significant $(\mathrm{p}<0.01)$ differences were still recovered.

15 It should be noted that our approach only estimates the expected average $\omega$ for each branch. This could be a source of error, particularly if functionally relevant mutations occur early or late in the history of a lineage ${ }^{5,42}$. Similarly, our method is ignorant to models that involve multiple predictions at different stages of a paralog's history. For example, He and Zhang's 
219 subneofunctionalization model predicts paralogs undergo a short subfunctionalization period 220 followed by sustained neofunctionalization ${ }^{53}$.

221 In conclusion, our results reveal distinct differences in patterns of substitution between

222 lineages descending from speciation and duplication events. $\omega$ ratios are on average more similar

223 between daughter lineages following speciation events than duplication events. This greater

224 amount of asymmetric evolution between paralogs appears to be driven by an increase in relaxed

225 selection in just one of the lineages. The maximum change from the parent branch is 5.6x greater

226 for paralogous lineages than orthologous lineages, whereas the minimum change from the parent 227 branch is nearly the same (0.98x) (Fig. 2B). Taken together these trends may indicate

228 neofunctionalization, where one copy retains the ancestral function while the other acquires a 229 new function, as the most dominant pattern for retained duplicated genes.

230

\section{Acknowledgements}

232 We would like to thank members of the Molette and Phyletica labs, in particular Damien Waits, 233 Caitlin Redak, Michael Tassia, and Dr. Yuanning Li for their feedback and comments. This is 234 Molette Biology Laboratory contribution \#XX, Auburn University Marine Biology Program 235 236 237

238 contribution \#XX, and Auburn University Museum of Natural History contribution \#XX.

1. Koonin, E. V. Orthologs, paralogs, and evolutionary genomics. Annu. Rev. Genet. 39, 309-

2. Dolinski, K. \& Botstein, D. Orthology and functional conservation in eukaryotes. Annu. Rev. Genet. 41, 465-507 (2007).

3. Studer, R. A. \& Robinson-Rechavi, M. How confident can we be that orthologs are similar, but paralogs differ? Trends in Genetics 25, 210-216 (2009).

5. Innan, H. \& Kondrashov, F. The evolution of gene duplications: classifying and distinguishing between models. Nature Reviews Genetics 11, 97 (2010). 
246 6. Hahn, M. W. Distinguishing among evolutionary models for the maintenance of gene 247 duplicates. Journal of Heredity 100, 605-617 (2009).

248 7. Ohno, S. Evolution by gene duplication. (Springer Science \& Business Media, 1970).

249 8. Force, A., Lynch, M., Pickett, F. B., Amores, A., Yan, Y. \& Postlethwait, J. Preservation of 250 duplicate genes by complementary, degenerative mutations. Genetics 151, 1531-1545 $251 \quad$ (1999).

252 9. Lynch, M. \& Conery, J. S. The evolutionary fate and consequences of duplicate genes. $253 \quad$ Science 290, 1151-1155 (2000).

254 10. Lynch, M. \& Katju, V. The altered evolutionary trajectories of gene duplicates. TRENDS in 255 Genetics 20, 544-549 (2004).

256 11. Hughes, A. L. The evolution of functionally novel proteins after gene duplication. 257 Proceedings of the Royal Society of London. Series B: Biological Sciences 256, 119-124 258 (1994).

259 12. Des Marais, D. L. \& Rausher, M. D. Escape from adaptive conflict after duplication in an 260 anthocyanin pathway gene. Nature 454, 762-765 (2008).

261 13. Gabaldón, T. \& Koonin, E. V. Functional and evolutionary implications of gene orthology. $262 \quad$ Nature Reviews Genetics 14, 360-366 (2013).

263 14. Fitch, W. M. Distinguishing homologous from analogous proteins. Systematic zoology 19, $26499-113(1970)$.

265 15. Kanehisa, M., Sato, Y., Kawashima, M., Furumichi, M. \& Tanabe, M. KEGG as a reference 266 resource for gene and protein annotation. Nucleic acids research 44, D457-D462 (2015). 
267 268

269

270

271

272

273

274

275

276

277

278

279

280

281

282

283

284

285

286

287

288

289

16. Thomas, P. D., Campbell, M. J., Kejariwal, A., Mi, H., Karlak, B., Daverman, R., Diemer, K., Muruganujan, A. \& Narechania, A. PANTHER: a library of protein families and subfamilies indexed by function. Genome research 13, 2129-2141 (2003).

17. Huerta-Cepas, J., Szklarczyk, D., Forslund, K., Cook, H., Heller, D., Walter, M. C., Rattei, T., Mende, D. R., Sunagawa, S. \& Kuhn, M. eggNOG 4.5: a hierarchical orthology framework with improved functional annotations for eukaryotic, prokaryotic and viral sequences. Nucleic acids research 44, D286-D293 (2015).

18. Consortium, U. UniProt: a worldwide hub of protein knowledge. Nucleic acids research 47, D506-D515 (2018).

19. Bleidorn, C. Phylogenomics. (Springer International Publishing, 2017).

20. Yanai, I., Graur, D. \& Ophir, R. Incongruent expression profiles between human and mouse orthologous genes suggest widespread neutral evolution of transcription control. Omics: $a$ journal of integrative biology 8, 15-24 (2004).

21. Liao, B.-Y. \& Zhang, J. Evolutionary conservation of expression profiles between human and mouse orthologous genes. Molecular biology and evolution 23, 530-540 (2005).

22. Nehrt, N. L., Clark, W. T., Radivojac, P. \& Hahn, M. W. Testing the ortholog conjecture with comparative functional genomic data from mammals. PLoS computational biology 7 , e1002073 (2011)

23. Chen, X. \& Zhang, J. The ortholog conjecture is untestable by the current gene ontology but is supported by RNA sequencing data. PLoS computational biology 8, e1002784 (2012).

24. Altenhoff, A. M., Studer, R. A., Robinson-Rechavi, M. \& Dessimoz, C. Resolving the ortholog conjecture: orthologs tend to be weakly, but significantly, more similar in function than paralogs. PLoS computational biology 8, e1002514 (2012). 
290

291

292

293

294

295

296

297

298

299

300

301

302

303

304

305

306

307

308

309

310

311

25. Thomas, P. D., Wood, V., Mungall, C. J., Lewis, S. E., Blake, J. A. \& Consortium, G. O. On the use of gene ontology annotations to assess functional similarity among orthologs and paralogs: a short report. PLoS computational biology 8, e1002386 (2012).

26. Rogozin, I. B., Managadze, D., Shabalina, S. A. \& Koonin, E. V. Gene family level comparative analysis of gene expression in mammals validates the ortholog conjecture. Genome biology and evolution 6, 754-762 (2014).

27. Kryuchkova-Mostacci, N. \& Robinson-Rechavi, M. Tissue-specificity of gene expression diverges slowly between orthologs, and rapidly between paralogs. PLoS computational biology 12, e1005274 (2016).

28. Dunn, C. W., Zapata, F., Munro, C., Siebert, S. \& Hejnol, A. Pairwise comparisons across species are problematic when analyzing functional genomic data. Proceedings of the National Academy of Sciences 115, E409-E417 (2018).

29. Vilella, A. J., Severin, J., Ureta-Vidal, A., Heng, L., Durbin, R. \& Birney, E. EnsemblCompara GeneTrees: Complete, duplication-aware phylogenetic trees in vertebrates. Genome research 19, 327-335 (2009).

30. Herrero, J., Muffato, M., Beal, K., Fitzgerald, S., Gordon, L., Pignatelli, M., Vilella, A. J., Searle, S. M., Amode, R. \& Brent, S. Ensembl comparative genomics resources. Database 2016, (2016).

31. Yang, Z. PAML 4: phylogenetic analysis by maximum likelihood. Molecular biology and evolution 24, 1586-1591 (2007).

32. Huerta-Cepas, J., Dopazo, H., Dopazo, J. \& Gabaldón, T. The human phylome. Genome biology 8, R109 (2007). 
312 33. Villanueva-Cañas, J. L., Laurie, S. \& Albà, M. M. Improving genome-wide scans of positive 313 selection by using protein isoforms of similar length. Genome biology and evolution 5, 457$314 \quad 467(2013)$.

315 34. Yang, Z. \& Bielawski, J. P. Statistical methods for detecting molecular adaptation. Trends in 316 ecology \& evolution 15, 496-503 (2000).

317 35. Yang, Z. Likelihood ratio tests for detecting positive selection and application to primate 318 lysozyme evolution. Molecular biology and evolution 15, 568-573 (1998).

319 36. Yang, Z. On the best evolutionary rate for phylogenetic analysis. Systematic Biology 47, $320 \quad 125-133(1998)$.

321 37. Hedges, L. V. Distribution theory for Glass's estimator of effect size and related estimators. 322 journal of Educational Statistics 6, 107-128 (1981).

323 38. Inman, H. F. \& Bradley Jr, E. L. The overlapping coefficient as a measure of agreement 324 between probability distributions and point estimation of the overlap of two normal densities. 325 Communications in Statistics-Theory and Methods 18, 3851-3874 (1989).

39. Massey Jr, F. J. The Kolmogorov-Smirnov test for goodness of fit. Journal of the American statistical Association 46, 68-78 (1951).

40. Seoighe, C., Johnston, C. R. \& Shields, D. C. Significantly different patterns of amino acid replacement after gene duplication as compared to after speciation. Molecular biology and evolution 20, 484-490 (2003).

41. Johnston, C. R., O’dushlaine, C., Fitzpatrick, D. A., Edwards, R. J. \& Shields, D. C. Evaluation of whether accelerated protein evolution in chordates has occurred before, after, or simultaneously with gene duplication. Molecular biology and evolution 24, 315-323 (2006). 
335 42. Han, M. V., Demuth, J. P., McGrath, C. L., Casola, C. \& Hahn, M. W. Adaptive evolution of 336 young gene duplicates in mammals. Genome research 19, 859-867 (2009).

337 43. Kondrashov, F. A., Rogozin, I. B., Wolf, Y. I. \& Koonin, E. V. Selection in the evolution of 338 gene duplications. Genome biology 3, research0008. 1 (2002).

339 44. Scannell, D. R. \& Wolfe, K. H. A burst of protein sequence evolution and a prolonged period 340 of asymmetric evolution follow gene duplication in yeast. Genome research 18, 137-147 $341 \quad$ (2008).

342 45. Francino, M. P. An adaptive radiation model for the origin of new gene functions. Nature 343 genetics 37, 573 (2005).

344 46. Conant, G. C. \& Wagner, A. Asymmetric sequence divergence of duplicate genes. Genome $345 \quad$ research $\mathbf{1 3}, 2052-2058(2003)$.

346 47. Kellis, M., Birren, B. W. \& Lander, E. S. Proof and evolutionary analysis of ancient genome 347 duplication in the yeast Saccharomyces cerevisiae. Nature 428, 617 (2004).

348 48. Brunet, F. G., Crollius, H. R., Paris, M., Aury, J.-M., Gibert, P., Jaillon, O., Laudet, V. \& 349 Robinson-Rechavi, M. Gene loss and evolutionary rates following whole-genome 350 duplication in teleost fishes. Molecular biology and evolution 23, 1808-1816 (2006).

351 49. Studer, R. A., Penel, S., Duret, L. \& Robinson-Rechavi, M. Pervasive positive selection on 352 duplicated and nonduplicated vertebrate protein coding genes. Genome research 18, 1393$3531402(2008)$.

354 50. Drummond, D. A., Bloom, J. D., Adami, C., Wilke, C. O. \& Arnold, F. H. Why highly 355 expressed proteins evolve slowly. Proceedings of the National Academy of Sciences 102, $356 \quad 14338-14343(2005)$. 
357 51. Cannarozzi, G. M. \& Schneider, A. Codon evolution: mechanisms and models. (Oxford $358 \quad$ University Press, 2012).

359 52. Stoletzki, N. \& Eyre-Walker, A. The Positive Correlation between d N/d S and d S in 360 Mammals Is Due to Runs of Adjacent Substitutions. Molecular biology and evolution 28, $361 \quad 1371-1380(2010)$.

362 53. He, X. \& Zhang, J. Rapid subfunctionalization accompanied by prolonged and substantial 363 neofunctionalization in duplicate gene evolution. Genetics 169, 1157-1164 (2005). 


\section{Figure 1}

\section{The Ortholog Conjecture}

A sample gene tree with duplication and speciation events. Genes that arose from a speciation event are orthologous to one another and genes that arose from a duplication event are paralogous. Under the Ortholog Conjecture, more changes are expected to occur along at least one of paralogous lineages following a duplication event. For each node we estimated the ratio of non-synonymous substitution rate $(\mathrm{dN})$ over synonymous substitution rate (dS) for the two daughter lineages. We then calculated the absolute difference between the two $(\Delta \omega)$ as well as the difference from the parent $\left(\Delta \omega_{p}\right)$ for an estimate of divergence. Under neofunctionalization we would expect to see more nonsynonymous substitutions (positive $\Delta \omega_{p}$ ) in one lineage (whichever one is acquiring a new function) while the other lineage remains the same (small $\Delta \omega_{\mathrm{p}}$ ), resulting in asymmetric selection between the two (large $\Delta \omega)$. Under conservation, both lineages are expected to maintain similar levels of nonsynonymous substitutions as in the parent lineage (small $\Delta \omega_{p}$ ), resulting in symmetric selection (small $\Delta \omega$ ). Under subfunctionalization more nonsynonymous substitutions are predicted in both daughter lineages $\left(\Delta \omega_{p}\right)$ as they partition aspects of the ancestral function. Subfunctionalization models generally do not make predictions regarding symmetry. 
$\omega_{\mathrm{p}}=\mathrm{dN} / \mathrm{dS}$ of parent branch

$\omega_{0}=\mathrm{dN} / \mathrm{dS}$ of one daughter branch

$\omega_{1}=\mathrm{dN} / \mathrm{d} S$ of other daughter branch
$\Delta \omega_{p 0}=\omega_{0}-\omega_{p}$ $\Delta \omega=\left|\omega_{0}-\omega_{1}\right|$

$\Delta \omega_{p 1}=\omega_{1}-\omega_{p}$

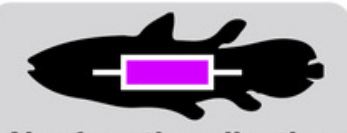

Neofunctionalization
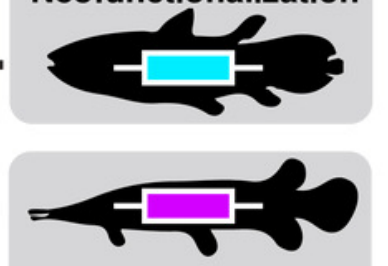

Conservation
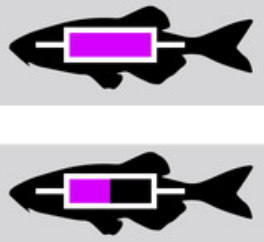

Subfunctionalization

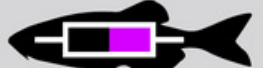

Speciations

Duplications
Expectation

$\Delta \omega_{\mathrm{p} 0} \approx 0$

$\Delta \omega>0$

$\Delta \omega_{\mathrm{p} 1}>0$

$\Delta \omega_{\mathrm{p} 0} \approx 0$

$\Delta \omega \approx 0$

$\Delta \omega_{\mathrm{p} 1} \approx 0$

$\Delta \omega_{\mathrm{p} 0}>0$

$\Delta \omega \gtrsim 0$

$\Delta \omega_{\mathrm{p} 1}>0$ 


\section{Figure 2}

$\omega$ estimates for orthologs and paralogs

A) kernel density plots of log transformed $\Delta \omega$ of orthologous and paralogous lineages. B)

$\Delta \omega_{\mathrm{p}}$ of orthologous and paralogous lineages with each pair of daughter lineages categorized by maximum and minimum values.
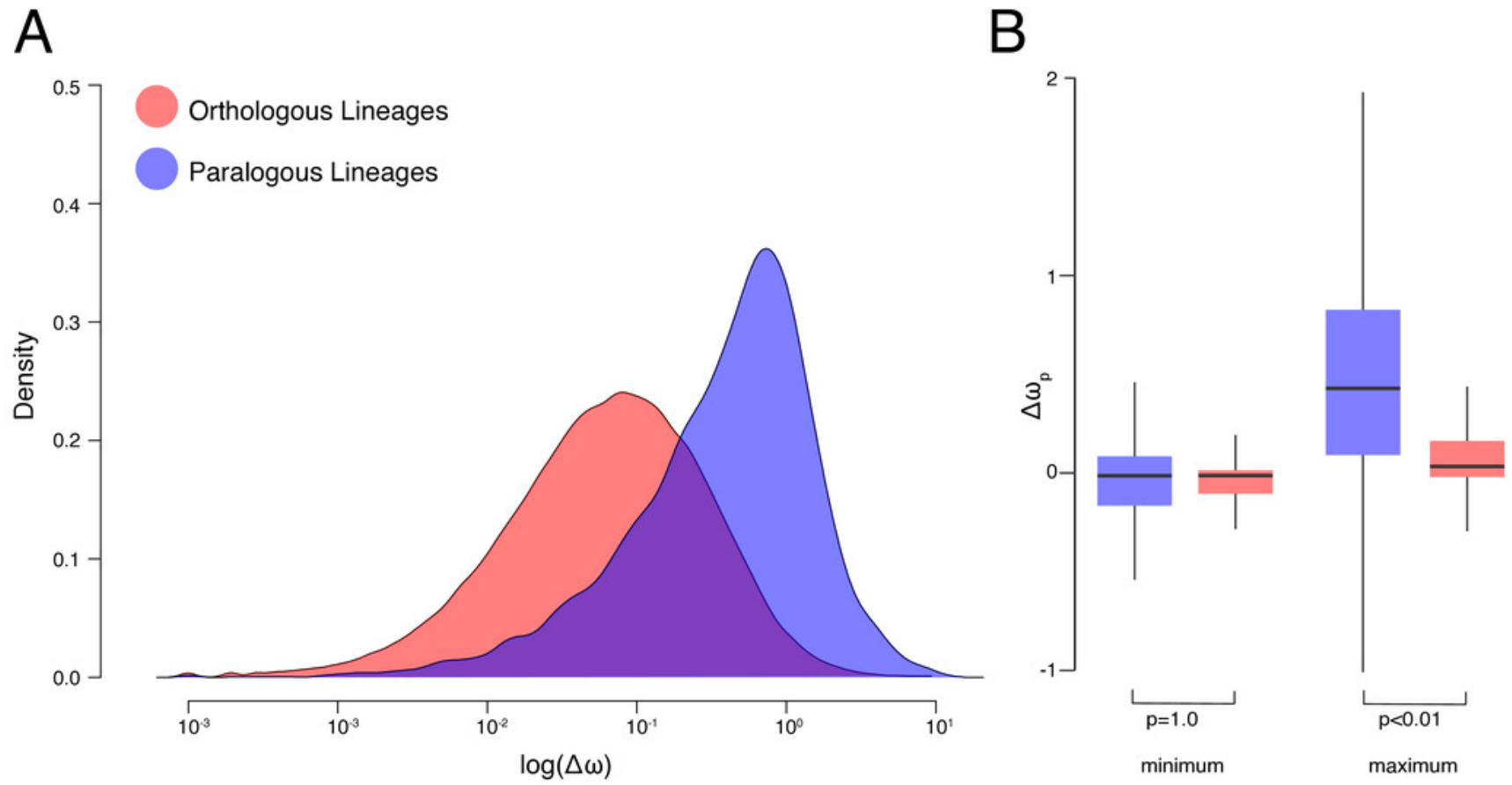
Figure 3

The Ortholog Conjecture through time

General additive model $95 \%$ confidence intervals of $\Delta \omega$ following speciation and duplication events over time, over the time-calibrated species tree of all taxa included in the study.

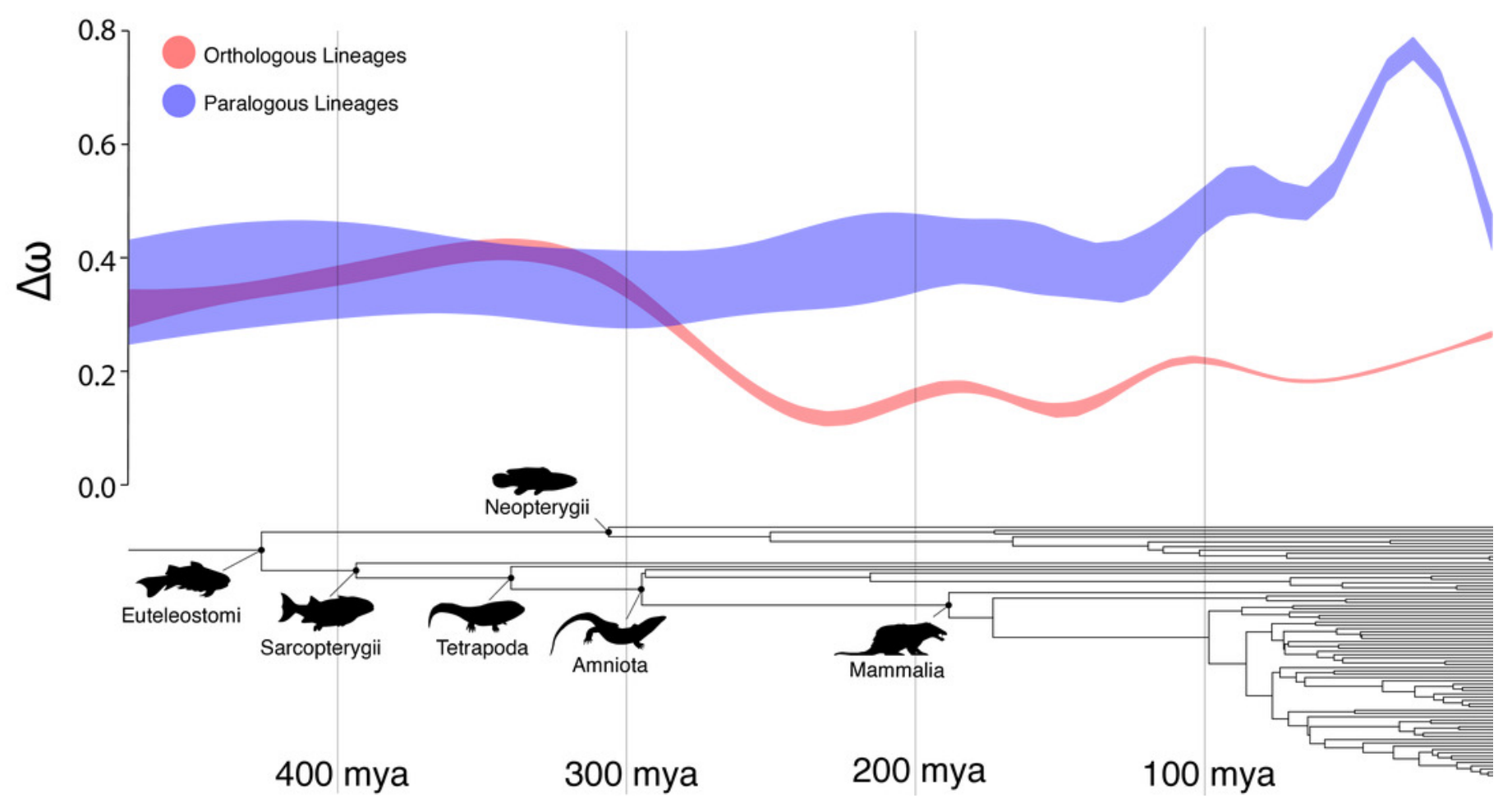

